Published in final edited form as:

Curr Hematol Malig Rep. 2013 September ; 8(3): 211-217. doi:10.1007/s11899-013-0163-4.

\title{
Hodgkin Lymphoma in Pregnancy
}

\author{
Veronika Bachanova and \\ University of Minnesota, Minneapolis, MN 55455, USA \\ Joseph M. Connors \\ BC Cancer Agency Centre for Lymphoid Cancer, 600 West 10th Avenue, Vancouver, BC, Canada \\ V5Z 4E6
}

\begin{abstract}
The peak incidence of Hodgkin lymphoma (HL) coincides with reproductive years, and as many as $3 \%$ of all $\mathrm{HL}$ patients present with concurrent pregnancy. The management of a pregnant patient with HL requires a multidisciplinary approach combining expertise in medical oncology, high-risk obstetrics, and neonatology, as well as effective communication with the patient and her family. The goal is to optimize the mother's chance of a cure while allowing for delivery of a healthy child. A pregnant patient with HL should be staged by clinical examination and judicious use of non-radiation imaging such as ultrasound, balancing the need for accurate disease assessment with the need to minimize invasive procedures. The treatment strategy is individualized to the symptoms, lymphoma stage, gestational age and the patients' wishes. Therapeutic options include treatment deferral or single-agent vinblastine with reservation of multi-agent chemotherapy until the second or third trimester for the small minority of patients with aggressive clinical presentation.
\end{abstract}

\section{Keywords}

Hodgkin lymphoma; Pregnancy; Chemotherapy

\section{Introduction}

Hodgkin lymphoma (HL) is the most common hematologic malignancy complicating pregnancy, with an estimated incidence of HL-associated deliveries of between 1:1,000 and 1:3,000 [1]. Although HL is more commonly diagnosed in women of low-parity and higher age, its biology, clinical course, and prognosis are otherwise similar to those seen in nonpregnant women. The medical challenge of concurrent HL and pregnancy stems from the need to manage the potentially life-threatening malignancy while giving the developing fetus the best chance of reaching term fully intact. Essentially, two patients need to be managed: one with lymphoma and the other without but potentially affected by the toxicity of any treatments. Religious, ethical, psychological, social and cultural beliefs, and attitudes of the patient and her partner, family and physicians all can affect decision-making. Thus, management of the disease and pregnancy not only involve the therapeutic approach, but 
also require attention to alleviating fear and anxiety and supporting the patient's emotional and social well-being. When HL is diagnosed during pregnancy, much of the discussion requires that the advising clinician balance the provision of expertise and knowledge about treatment options and prognosis with respect for ethical principles, compassion and acceptance of patient autonomy.

The clinical data comparing the effectiveness of treatment approaches for concurrent HL and pregnancy are limited to case-control series, retrospective reports of small numbers of patients and expert opinions. Nevertheless, we believe that the available evidence suggests that when $\mathrm{HL}$ is discovered during pregnancy, it is almost always possible to control the lymphoma and allow the pregnancy to continue to full term. The best results are achieved under the direction of a multidisciplinary team composed of a hemato-oncologist knowledgeable in the treatment of $\mathrm{HL}$, an obstetrician experienced in the management of high-risk pregnancy, a pediatrician/ neonatologist familiar with hematologic problems in the neonate, and a nurse coordinator who augments the communication and delivery of care. Decision-making must therefore be guided by a judicious mix of careful clinical judgment, the experience of involved team members, knowledge of the natural history of $\mathrm{HL}$ and consideration of the patient's personal beliefs and desires [2-4].

\section{Diagnostic Approach to HL During Pregnancy}

Planning the diagnostic evaluation of $\mathrm{HL}$ in a pregnant patient does not require complete staging, but it should explain patient symptoms and estimate lymphoma stage. The guiding diagnostic principle should be to carefully balance accurate disease assessment with the need to minimize the use of invasive procedures. The histopathologic diagnosis of HL should be based on tissue examination obtained by excisional or incisional tissue biopsy. The most common subtype encountered in pregnancy is nodular sclerosing HL. The initial evaluation should include a complete history and physical examination with careful documentation of B symptoms and palpation of node-bearing areas and the abdomen. Standard laboratory tests should include hemoglobin, complete differential white blood cell count, platelet count, erythrocyte sedimentation rate (ESR), liver and renal function assessment, lactate dehydrogenase and serum protein electrophoresis including albumin level. Some diagnostic tests, particularly ESR and alkaline phosphatase, can be increased in pregnancy and, therefore must be interpreted carefully.

Radiologic staging should be limited to the minimum necessary to identify disease that seriously threatens the immediate well-being of mother or child. Combined F-18 fluorodeoxyglucose (FDG) positron emission tomography (PET)/computed tomography (CT) scan is a standard imaging modality for staging HL under ordinary circumstances, but it employs ionizing radiation that is potentially harmful to an unborn fetus. In a recent case report, the fetal radiation doses during PET scan were as high as $11.8 \mathrm{mGy}$; CT scan added a further $10 \mathrm{mGy}$, suggesting that the use of PET/CT scan should be avoided in pregnancy [5•]. A single postero-anterior radiograph of the chest, with proper shielding, should be obtained to characterize the extent of mediastinal and pulmonary disease, because overall radiation exposure is much lower than the dose required to cause malformation during organogenesis [6]. Abdominal ultrasonography should be used to identify the extent and size 
of retroperitoneal nodal disease and provides sufficient detail for proper management [3]. Magnetic resonance imaging (MRI) without use of gadolinium can also be used and is, at least theoretically, free of potential toxicity to the fetus [7]; however, the amount of detail provided in excess of what can be found with ultrasonography is unnecessary and the safety of the intensive magnetic fields required is not fully established.

Additional assessments can include bone marrow biopsy for patients with B symptoms or abnormalities in blood counts such as anemia, thrombocytopenia, or leucopenia, and focused otolaryngologic examination in patients with upper cervical lymphadenopathy. For those patients in whom chemotherapy is planned, echocardiography may be used to assess left ventricular function. The goal of clinical and radiologic staging is to provide guidance about disease pace, and to evaluate whether treatment can be deferred or whether disease is symptomatic or impacts organ function and therefore requires immediate therapy. Hence, tests should only be performed if decisions regarding immediate management will be influenced.

\section{Treatment of HL During Pregnancy}

A multidisciplinary team of experts working together is required to develop an individualized management plan (Table 1). The therapeutic options for pregnant patients with HL depend on presenting features, gestational age at diagnosis, fetal risks and the patient's wishes regarding the continuation of pregnancy. What little we know about managing pregnant HL patients comes from several small series of cases and anecdotal descriptions. However, this limited evidence can provide useful guidance when complemented by careful clinical judgment and knowledge of the natural history of HL. The clinical challenge lies in determining the effect of treatment delay on maternal survival versus the risk of unintended abortion, fetal malformation and adverse perinatal outcomes associated with the use of chemotherapy and radiotherapy. Frequent communication with the patient and her family is crucial to ensure understanding and alleviate anxiety and fear.

In the largest clinical series reported to date, 48 pregnant women were matched to nonpregnant controls with $\mathrm{HL}$, and this study showed that stage and clinical presentation, course of disease, response to therapy and long term outcomes were not impacted by pregnancy [8]. The overall survival rate was similar when compared with age-equivalent and stageequivalent non-pregnant controls $[8,9]$. In addition, two reports found no difference in survival among women who did not have a therapeutic abortion in comparison to those who did $[10,11]$. Several authors have observed that HL by itself does not appear to have an adverse effect on the course of pregnancy, fetal development, labor or puerperium [12, 13]. Therefore, therapeutic abortion is not necessary to ensure the best prognosis.

\section{General Therapeutic Principles}

Most patients with $\mathrm{HL}$ and concomitant pregnancy require no immediate intervention. As a general rule, any treatment, such as radiation or chemotherapy, should be avoided during the first trimester unless severe symptoms are present or organ function is seriously compromised. Almost all chemotherapy agents have been documented to be teratogenic in 
animals or humans, although for some drugs only experimental data exist. Chemotherapy during the first trimester may increase the risk of spontaneous abortion, fetal death, and major malformation; the fetus is extremely vulnerable from the second to eighth week of gestation, during which time organogenesis occurs. Even after primary organogenesis, several organs, including the eyes, genitalia, hematopoietic system, and central nervous system, remain vulnerable to chemotherapy and radiation therapy. Although only very rarely necessary, the option of pregnancy termination needs to be considered for a patient whose first trimester is complicated by severely symptomatic or life-threatening disease, where the need for immediate chemotherapy may affect fetal development. The medical team needs to consider the often underestimated impact of pregnancy termination on woman's subsequent well-being. A recent population-based study of 455 women younger than 40 years of age with history of cancer (including HL patients) were interviewed and screened for long-term distress and quality of life related to cancer-related infertility. Childless women were the most distressed, and even at long-term follow-up, the distress over interrupted childbearing persisted and impacted psychosocial and mental health [14].

\section{Early Stage HL During Pregnancy}

The majority of HL patients diagnosed during pregnancy have stage IA or IIA disease and are asymptomatic or minimally symptomatic. Treatment for these patients can be deferred, but close monitoring and follow-up through the entire pregnancy has to be ensured. In the Stanford series, 11 out of 17 patients required no treatment for HL concomitant with pregnancy [15]. The approach of watchful waiting has also been demonstrated to be safe in a small case series of 19 patients from Royal Mardsen Hospital [16]. Many patients can be monitored throughout pregnancy until normal full term delivery without treatment for lymphoma. Nevertheless, therapy is required if severe symptoms or organ dysfunction develops. Patients with stage IA-IIA HL with localized or stable disease who have chemotherapy safely deferred can complete appropriate staging and initiate treatment soon after delivery. In two recent studies, among HL patients opting to delay treatment until after delivery, the birth weight, mean gestational age, and method of delivery was similar to normal pregnancies $[8,13]$.

Based primarily on experience acquired prior to the development of highly effective chemotherapy, several studies demonstrated the efficacy of irradiation for symptomatic patients with cervical adenopathy staged IB or IIB, or patients with respiratory symptoms due to enlarging mediastinal masses. However, at most, radiation should be reserved for cases where it is absolutely necessary and extreme caution should be taken to provide special shielding of the fetus with ten half-value layer shields $[8,10,17,18]$. Prior to treatment, the maximal dose to the fetus should be calculated and then monitored throughout the therapy. An inverted $\mathrm{Y}$ field is not an option at any time during pregnancy. In a recent report, the estimate of scattered dose to conceptus from involved-field radiotherapy on lineal accelerator associated with cervical node irradiation was below the threshold of $10 \mathrm{cGy}$; however, radiation therapy to lymph nodes in the axilla, mediastinum, and neckmediastinum could lead to a dose of $>10 \mathrm{cGy}$ and therefore should not be recommended in the first trimester $[19,20]$. It is important to recall that use of any therapeutic radiation during pregnancy, especially in advanced gestational age, results in direct or scattered fetal 
exposure. The effects of fetal irradiation may become evident only many years later. For example, a known risk for the fetus from radiation in the second half of gestation is acquisition of blood dyscrasias or leukemia later in life [21]. In addition, irradiation encompassing the mediastinum exposes breast tissue to scatter radiation and potentially increases the risk of later secondary breast cancer and other secondary malignancies [22].

Because radiation unnecessarily endangers the fetus, a better choice, if treatment is necessary, is systemic chemotherapy. If intervention is required, especially after the first trimester, selected symptomatic patients can be treated with single agent vinblastine or modified multiagent chemotherapy regimens, which have been used frequently in the second and third trimester, with favorable outcomes for both mother and newborn [3, 4]. What is known about the effects of chemotherapy is drawn from several small clinical series and anecdotal descriptions. [8-11, 13, 15, 16, 18, 23, 24]. Chemotherapy use in the first trimester, especially with multiple agents, alkylating agents and anti-metabolites, may be associated with fetal malformations and increased risk of spontaneous abortions and stillbirths. Therefore, single-agent vinblastine used as monotherapy appears to be the preferred agent if a patient requires treatment, because it does not cross the placenta and has been safely used in patients in all trimesters [10, 25-27].

\section{Treatment Strategy for Advanced HL in Pregnancy}

Management during pregnancy of HL with bulky disease, visceral involvement, B symptoms, sub-diaphragmatic disease, or rapid disease progression remains controversial. Several observers have reported increased risk of spontaneous abortion and fetal malformations with alkylating agents such as mechlorethamine, cyclophosphamide, procarbazine, and chlorambucil; anti-metabolites such as methotrexate; or multi-agent regimens such as MOPP (mechlorethamine, vincristine, prednisone and procarbazine) and MOP. These classes of agents should be avoided during pregnancy because of known carcinogenicity and teratogenicity $[8,9,15,16,23,24]$ However, ABVD (doxorubicin, bleomycin, vinblastine, and dacarbazine), the current standard of care in North America, has been used during pregnancy, and the limited experience that has been reported has not identified obvious negative effects on the fetus, although the number of patients is still small and whether the trimester of exposure is important is unclear [18, 23, 27, 28, 29]. In a small series of 26 children with HL with a long follow-up of 3-19 years, children born to women who received chemotherapy for HL in the second and third trimesters delivered healthy newborns without short-term or long-term neurological, developmental, or infectious complication or secondary malignancies [13]. However, the use of anthracyclines at doses exceeding $70 \mathrm{mg} / \mathrm{m} 2$ per cycle has been associated with a 30-fold increase in severe fetal toxicity including death, malformations, and cardiac toxicity. The ABVD regimen contains doxorubicin at a lower dosage ( $25 \mathrm{mg} / \mathrm{m}^{2}$ with each dose of ABVD); however, caution and careful counseling is always required when ABVD is administered in the second and third trimester. For example, one series reported stillbirth of twins in an HL patient who started the ABVD regimen at 14 weeks of gestation [29]. In addition, multiagent chemotherapy used in the last trimester of pregnancy may often result in prematurity, lower birth weights, and neonatal myelosuppression [30•]. In a recent European series of 176 neonates born to mothers with malignancy, of which 13 had HL, binomial testing revealed a significant 
increase in small-for-gestational-age children in the group receiving treatment during pregnancy versus those not treated during pregnancy [31••]. Nevertheless, some recent reports suggest that ABVD used even in the first trimester for patients with HIV-associated HL may be associated with favorable outcomes for the patient and fetus [32,33]. Data on the use of more intensive regimens such as Stanford V (doxorubicin, vinblastine, mechlorethamine, etoposide and prednisone) or BEACOPP (bleomycin, etoposide, doxorubicin, cyclophosphamide, vincristine, prednisone and procarbazine) during pregnancy are not available; however, because both contain alkylating agents, they should be avoided.

Rather than expose the fetus to the potential adverse effects of multiple agents, an alternative approach employing single agent chemotherapy should be considered for symptomatic disease. Vinblastine was first described for this use more than 40 years ago [25, 34], and is a particularly attractive agent because of its high level of effectiveness against HL in treatment-naïve patients (>75\% response rate) and modest acute toxicity. Although teratogenic effects have been reported in mice, neither teratogenic nor carcinogenic effects are apparent in humans at doses therapeutic for lymphoma. The combination of a high level of effectiveness, minimal acute toxicity and low likelihood of a negative effect on the fetus make vinblastine an attractive agent to suppress HL during pregnancy. Infrequent doses at intervals of several weeks or longer can be given to control HL until delivery at term, minimizing risks to mother and child. Progression despite vinblastine, which occurs infrequently, should be treated with full-dose ABVD, because evidence of chemotherapy resistance signifies aggressive disease requiring multiagent chemotherapy. Standard dosing of $6 \mathrm{mg} / \mathrm{m}^{2}$ is unlikely to cause significant myelosuppression, but careful timing to avoid a blood cell count nadir near delivery is prudent.

We have managed 18 pregnant patients with coincident HL at the British Columbia Cancer Agency over the past 23 years using the approach described above and in Fig. 1. Eleven patients remained off treatment through term delivery, and six required vinblastine to control disease. Fourteen of the 18 patients are still alive and well and four have died, two from HL and one each from acute myeloid leukemia and a retroperitoneal sarcoma. All 18 delivered normal children who now range in age from 2 to 23 years (median 17). Although these children have not been systematically assessed, no overt abnormality has become apparent [3]. Management built around conservative use of single agent vinblastine has allowed normal term delivery of the children and effective management of the mother's HL with a minimum of psychological stress, and appears to be a reasonable approach to this rare problem of coincident pregnancy and HL.

\section{Planning the Delivery and Managing the Post-Partum Period in Patients with HL}

Post-delivery oncologic care is a critical step in managing HL in pregnancy. Patients with $\mathrm{HL}$ in whom gestation progressed to term will need team members to plan the timing and mode of delivery. Fetal maturity should preferably be the criterion to induce delivery. In a multidisciplinary setting, a maximal effort should be made to delay delivery until at least 35 to 37 weeks. Pathologic examination of the placenta should be performed after birth, 
because this has been a site of documented, albeit exceedingly rare, metastases [35]. A recent observational large series of patients with cancer diagnosed during pregnancy demonstrated that over $54 \%$ of neonates exposed to chemotherapy in their second and third trimester were born preterm, with a subsequently high rate of admission to the neonatal intensive care unit. In the vast majority $(89.7 \%)$, the delivery was iatrogenically induced and complications were mostly related to pre-maturity $[31 \bullet, 36 \bullet$. Thus, preterm delivery should be avoided if possible. A coordinated, detailed peripartum plan developed by the neonatologist, obstetrician with experience in high-risk pregnancies and oncologist/ hematologist is required to minimize complications. Breast feeding must be discouraged in those patients who continue chemotherapy postpartum, as most cytotoxic agents can be excreted into the breast milk. In the perinatal period, patients who had received any therapy for HL during the pregnancy should be fully restaged after delivery, including PET/CT scanning. Patients treated with radiation, single-agent vinblastine or other chemotherapy during the pregnancy can no longer be accurately staged, and therefore should be treated with a full course of six to eight cycles of multiagent chemotherapy. Post-treatment PET/CT imaging has a strong predictive value for overall survival and should be considered to assess the depth of post-therapy remission.

\section{Relapsed HL and Concomitant Pregnancy}

Occasionally, the patient with history of HL presents with relapsed lymphoma and concurrent pregnancy. There are limited data to guide the therapeutic decisions for such a rare clinical situation; however, care can be guided by principles similar to those recommended for newly diagnosed HL and concurrent pregnancy. Individualized recommendations will depend on the initial HL stage, type of primary therapy used in the past, and the time from remission to relapse, as well as current symptoms, stage, and gestational age. Patients with low disease burden in second or third trimester can often be managed by careful watching. Most patients who relapse with advanced HL or those who had received prior chemotherapy will eventually be considered for treatment with salvage multiagent chemotherapy, followed by high-dose myeolablative chemotherapy and autologous hematopoietic stem cell rescue. Nevertheless, conservative management that allows the pregnancy to develop to term is often possible, and interventions for definite therapy, such as autologous stem cell transplant, can be deferred until soon after delivery. The decision of when to initiate treatment rests on careful and frequent monitoring of the patient and the pace of the disease. If rapidly symptomatic disease develops in the first trimester, planned pregnancy interruption and subsequent standard treatment should be considered. The coordination of care with transplant physician and his team is necessary to ensure timely post-delivery interventions.

\section{Conclusions}

The diagnostic and therapeutic approach to the patient with concurrent HL and pregnancy presents the challenge of managing two lives. The goal is to give the mother with HL the best chance for cure while preserving the healthy development of the fetus. The best balanced strategy is achieved by careful assessment of HL, particularly with respect to disease symptoms and gestational age. Usually, diagnostic tests can be limited to non- 
invasive imaging. PET/CT should be avoided during pregnancy. Treatment can often be deferred, and if intervention is needed to control symptoms or prevent organ dysfunction, intermittent single agent vinblastine is a preferred choice. In rare cases, symptomatic HL in early gestational age or with rapidly progressive disease during the first trimester may be best managed by pregnancy termination followed by standard multiagent therapy. Chemotherapy with ABVD can be carefully considered for those symptomatic HL patients in second and third trimester whose disease has progressed despite single agent vinblastine. A team composed of a hematologist, an obstetrician, a neonatologist and a nurse with experience in complicated obstetrical care is required to ensure comprehensive management of coincident $\mathrm{HL}$ and pregnancy. Furthermore, establishment of a prospective central registry for patients with concurrent HL and pregnancy to allow data collection on long-term follow-up of children born to HL patients would enhance the care of patients with this uncommon complication of pregnancy and that of their children, by providing a larger database of relevant information than is currently available.

\section{References}

Papers of particular interest, published recently, have been highlighted as:

- Of importance

•- Of major importance

1. Smith LH, Danielsen B, Allen ME, Cress R. Cancer associated with obstetric delivery: results of linkage with the California cancer registry. Am J Obstet Gynecol. 2003; 189:1128-35. [PubMed: 14586366]

2. Connors JM. Clinical manifestations and natural history of Hodgkin's lymphoma. Cancer J. 2009; 15:124-8. [PubMed: 19390307]

3. Connors JM. Challenging problems: coincident pregnancy, HIV infection, and older age. Hematol Am Soc Hematol Educ Program. 2008:334-9.

4. Bachanova V, Connors JM. How is Hodgkin lymphoma in pregnancy best treated? ASH evidencebased review 2008. Hematol Am Soc Hematol Educ Program. 2008:33-4.

5•. Zanotti-Fregonara P, Jan S, Taieb D, et al. Absorbed 18F-FDG dose to the fetus during early pregnancy. J Nucl Med. 2010; 51:803-5. Impact of PET/CT imaging on pregnancy. [PubMed: 20395321]

6. O'Connor SJ, Verma H, Grubnic S, Rayner CF. Chest radiographs in pregnancy. BMJ. 2009; 339:b4057. [PubMed: 19819929]

7. Nicklas AH, Baker ME. Imaging strategies in the pregnant cancer patient. Semin Oncol. 2000; 27:623-32. [PubMed: 11130469]

8. Lishner M, Zemlickis D, Degendorfer P, et al. Maternal and foetal outcome following Hodgkin's disease in pregnancy. Br J Cancer. 1992; 65:114-7. [PubMed: 1733434]

9. Gobbi PG, Attardo-Parrinello A, Danesino M, Motta C, Di Prisco AU, Rizzo SC, et al. Hodgkin's disease and pregnancy. Haematologica. 1984; 69:336-41. [PubMed: 6432646]

10. Nisce LZ, Tome MA, He S, et al. Management of coexisting Hodgkin's disease and pregnancy. Am J Clin Oncol. 1986; 9:146-51. [PubMed: 3717081]

11. Jacobs C, Donaldson SS, Rosenberg SA, Kaplan HS. Management of the pregnant patient with Hodgkin's disease. Ann Intern Med. 1981; 95:669-75. [PubMed: 7305142]

12. Barry RM, Diamond HD, Craver LF. Influence of pregnancy on the course of Hodgkin's disease. Am J Obstet Gynecol. 1962; 84:445-54. [PubMed: 13865327] 
13. Aviles A, Diaz-Maqueo JC, Talavera A, et al. Growth and development of children of mothers treated with chemotherapy during pregnancy: current status of 43 children. Am J Hematol. 1991; 36:243-8. [PubMed: 1707227]

14. Canada AL, Schover LR. The psychosocial impact of interrupted childbearing in long-term female cancer survivors. Psychooncology. 2012; 21:134-43. [PubMed: 22271533]

15. Gelb AB, van de Rijn M, Warnke RA, Kamel OW. Pregnancy-associated lymphomas. A clinicopathologic study. Cancer. 1996; 78:304-10. [PubMed: 8674008]

16. Thomas PR, Biochem D, Peckham MJ. The investigation and management of Hodgkin's disease in the pregnant patient. Cancer. 1976; 38:1443-51. [PubMed: 953978]

17. Byram D, Foulstone P. Radiotherapy for Hodgkin's disease in pregnancy. Australas Radiol. 1997; 41:407-8. [PubMed: 9409040]

18. Anselmo AP, Cavalieri E, Enrici RM, et al. Hodgkin's disease during pregnancy: diagnostic and therapeutic management. Fetal Diagn Ther. 1999; 14:102-5. [PubMed: 10085508]

19. Mazonakis M, Lyraraki E, Varveris C, et al. Conceptus dose from involved-field radiotherapy for Hodgkin's lymphoma on a linear accelerator equipped with MLCs. Strahlenther Onkol. 2009; 185:355-63. [PubMed: 19506818]

20. Friedman E, Jones GW. Fetal outcome after maternal radiation treatment of supradiaphragmatic Hodgkin's disease. CMAJ. 1993; 149:1281-3. [PubMed: 8221483]

21. Latourette HB. Induction of lymphoma and leukemia by diagnostic and therapeutic irradiation. Radiol Clin N Am. 1968; 6:57-61. [PubMed: 5652935]

22. Ng AK, Bernardo MV, Weller E, et al. Second malignancy after Hodgkin disease treated with radiation therapy with or without chemotherapy: long-term risks and risk factors. Blood. 2002; 100:1989-96. [PubMed: 12200357]

23. Ebert U, Loffler H, Kirch W. Cytotoxic therapy and pregnancy. Pharmacol Ther. 1997; 74:207-20. [PubMed: 9336023]

24. Tawil E, Mercier JP, Dandavino A. Hodgkin's disease complicating pregnancy. J Can Assoc Radiol. 1985; 36:133-7. [PubMed: 4019555]

25. Rosenzweig AI, Crews QE Jr, Hopwood HG. Vinblastine sulfate in Hodgkin's disease in pregnancy. Ann Intern Med. 1964; 61:108-12. [PubMed: 14175829]

26. Lacher MJ, Geller W. Cyclophosphamide and vinblastine sulfate in Hodgkin's disease during pregnancy. JAMA. 1966; 195:486-8. [PubMed: 5951840]

27. Dilek I, Topcu N, Demir C, et al. Hematological malignancy and pregnancy: a single-institution experience of 21 cases. Clin Lab Haematol. 2006; 28:170-6. [PubMed: 16706933]

28. Cardonick E, Iacobucci A. Use of chemotherapy during human pregnancy. Lancet Oncol. 2004; 5:283-91. [PubMed: 15120665]

29. Fanale, MA., Lai, C-M., Rimes, SA., et al. Presented at Annual American Society of Hematology meeting. Salt Lake City, USA: Dec 8-12. 2012 Positive maternal-fetal outcomes with treatment of lymphoma during pregnancy: UT MD Anderson Cancer Prospective Experience.

30•. Anatolian Group AMOS. Ustaalioglu BB, Gumus M, et al. Malignancies diagnosed during pregnancy and treated with chemotherapy or other modalities (review of 27 cases): multicenter experiences. Int J Gynecol Cancer. 2011; 20:698-703. Multicenter case series of recent date.

31••. Van Calsteren K, Heyns L, De Smet F, et al. Cancer during pregnancy: an analysis of 215 patients emphasizing the obstetrical and the neonatal outcomes. J Clin Oncol. 2010; 28:683-9. The paper presents neonatal and obstetrics outcomes of patients with invasive cancer diagnosed during pregnancy collected of over 20 years in international collaborative setting. [PubMed: 19841323]

32. Klepfish A, Schattner A, Shtalrid M, et al. Advanced Hodgkin's disease in a pregnant HIV seropositive woman: favorable mother and baby outcome following combined anticancer and antiretroviral therapy. Am J Hematol. 2000; 63:57-8. [PubMed: 10602171]

33. Okechukwu CN, Ross J. Hodgkin's lymphoma in a pregnant patient with acquired immunodeficiency syndrome. Clin Oncol (R Coll Radiol). 1998; 10:410-1. [PubMed: 9890547]

34. Armstrong JG, Dyke RW, Fouts PJ. Vinblastine sulfate treatment of Hodgkin's disease during a pregnancy. Science. 1964; 143:703. [PubMed: 14081247] 
35. Dildy GA 3rd, Moise KJ Jr, Carpenter RJ Jr, Klima T. Maternal malignancy metastatic to the products of conception: a review. Obstet Gynecol Surv. 1989; 44:535-40. [PubMed: 2544836]

36•. Amant F, Van Calsteren K, Halaska MJ, et al. Long-term cognitive and cardiac outcomes after prenatal exposure to chemotherapy in children aged 18 months or older: an observational study. Lancet Oncol. 2012; 13:256-64. This paper present the multicenter observational cohort study recording the general health, cardiac function, and neurodevelopmental outcomes of children who were prenatally exposed to chemotherapy. [PubMed: 22326925] 


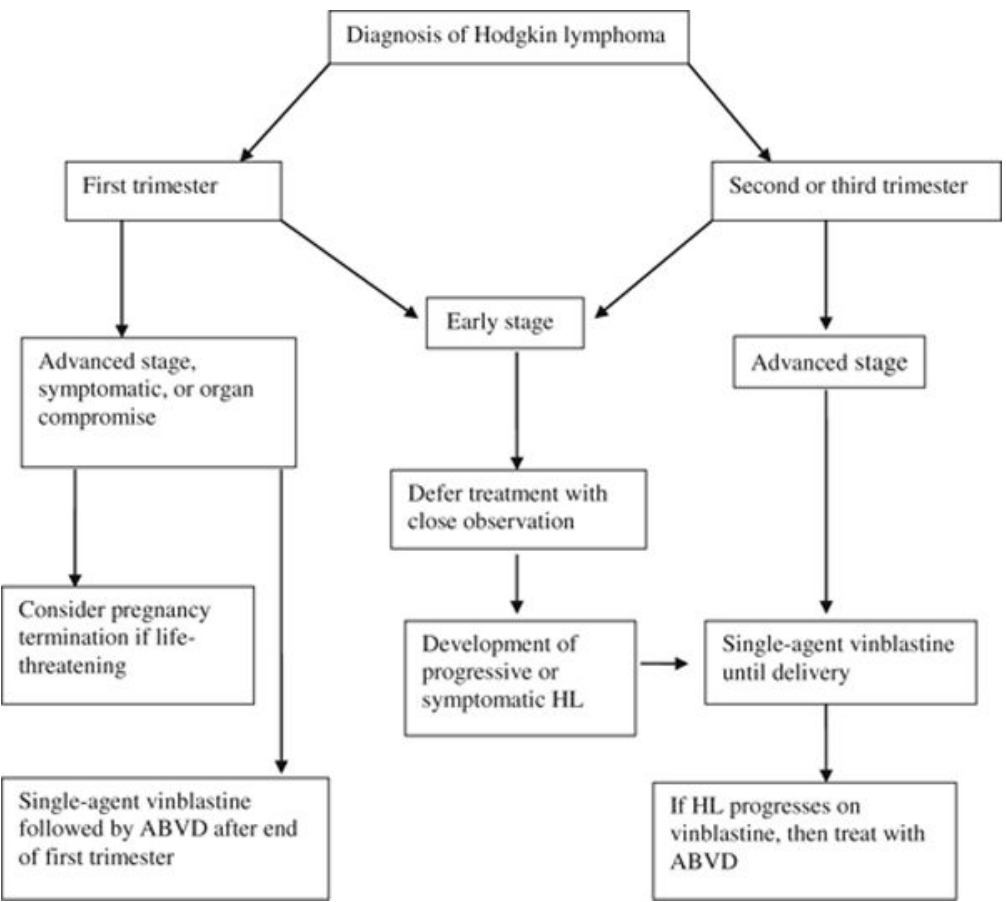

Fig. 1.

Recommended algorithm for treatment of pregnancy-associated Hodgkin lymphoma (HL) ABVD doxorubicin, bleomycin, vinblastine, dacarbazine 


\section{Table 1}

Characteristics of an ideal multidisciplinary team treating the pregnant patient with concomitant Hodgkin lymphoma

\begin{tabular}{|c|c|}
\hline \multirow[t]{5}{*}{ Obstetrician } & Usually makes the diagnosis, referral to heme/onc \\
\hline & Brings experience in high-risk pregnancies (patients with active malignancy) \\
\hline & Provides counseling regarding pregnancy termination (if recommended by the team) \\
\hline & Establishes the timing and method of delivery \\
\hline & Supervises effective postpartum contraception for a minimum of 2 years (greatest risk of relapse) \\
\hline \multirow[t]{12}{*}{ Hematologist/medical oncologist } & Performs oncologic history and physical and plans staging \\
\hline & $\begin{array}{l}\text { History searching for B symptoms or other symptomatic problems suggesting more advanced } \\
\text { disease }\end{array}$ \\
\hline & O Physical examination for lymphadenopathy or organomegaly \\
\hline & O Complete blood cell counts \\
\hline & $\begin{array}{l}\text { O Serum creatinine, alkaline phosphatase, lactate dehydrogenase, bilirubin and protein } \\
\text { electrophoresis (including albumin level) }\end{array}$ \\
\hline & O Chest radiograph, PA view only, with appropriate shielding \\
\hline & O Abdominal ultrasound for retroperitoneal lymphadenopathy \\
\hline & Formulates therapeutic plan \\
\hline & Administers chemotherapy if deemed necessary \\
\hline & $\begin{array}{l}\text { Provides supportive care for patients treated with chemotherapy to keep } \mathrm{Hgb} \geq 100 \mathrm{~g} / \mathrm{L} \text { and platelet } \\
\text { count } \geq 30 \times 10^{9} / \mathrm{L} \text {, and reviews safety of medications used for supportive care during pregnancy }\end{array}$ \\
\hline & $\begin{array}{l}\text { Coordinates delivery planning and chemotherapy administration to ensure that platelet count is } \\
\geq 50 \times 10^{9} / \mathrm{L} \text { at the time of delivery }\end{array}$ \\
\hline & Arranges oncology follow-up after pregnancy to complete appropriate staging \\
\hline \multirow[t]{8}{*}{ Neonatologist } & Has experience in high-risk pregnancies \\
\hline & Has experience in childhood hematologic disorders \\
\hline & Examines placenta and arranges histopathologic evaluation for presence of metastasis \\
\hline & Coordinates newborn care at time of delivery \\
\hline & Delivers early post-natal care of newborn \\
\hline & Registers newborn to central registry of children born to pregnant mothers with HL \\
\hline & Counsels about breastfeeding \\
\hline & Schedules long term follow-up of newborn \\
\hline \multirow[t]{2}{*}{ Nurse coordinator } & Coordinates communication among subspecialists \\
\hline & Helps interpret complex communication with the patient \\
\hline
\end{tabular}

Curr Hematol Malig Rep. Author manuscript; available in PMC 2017 November 21. 